\title{
A Unique Case of Intranasal Metastasis from Occult Poorly Differentiated Thyroid Carcinoma
}

\author{
Rauf Oğuzhan Kum ${ }^{1}$, Erdinç Aygenç², Battal Tahsin Somuk ${ }^{3}$, Pelin Börcek ${ }^{4}$ Cafer Özdem ${ }^{5}$ \\ ${ }^{1}$ Clinic of Ear, Nose and Throat, Ankara Numune Training and Research Hospital, Ankara, Turkey \\ ${ }^{2}$ Clinic of Ear, Nose and Throat, Ankara Güven Hospital, Ankara, Turkey \\ ${ }^{3}$ Department of Otorhinolaryngology, Gaziosmanpaşa University Faculty of Medicine, Tokat, Turkey \\ ${ }^{4}$ Clinic of Pathology, Ankara Numune Training and Research Hospital, Ankara, Turkey \\ ${ }^{5}$ Clinic of Ear, Nose and Throat, Ankara MedArt Hospital, Ankara, Turkey
}

Background: Poorly differentiated thyroid carcinomas (PDTCs) lie, both morphologically and behaviorally, between well-differentiated and undifferentiated carcinomas. Metastasis of poorly differentiated thyroid carcinoma to the intranasal cavity has not been reported previously in the literature.

Case Report: A 48-year-old male patient presented with massive epistaxis and nasal obstruction. On nasal examination, a bleeding, vascular mass was seen filling the left nasal cavity. The histopathological report of the nasal mass was well-differentiated thyroid carcinoma metastasis. Whole body scintigraphy, ultrasonography and positron emission tomography were done to rule out other possible metastases in the body and determine the origin of the tumor, which was identified as the left lobe of the thyroid gland, and there were multiple metastases involving the lung, sacroiliac area, and left humerus. Histopathological examination of a thyroidectomy specimen revealed PDTC consisting of insular, follicular, and papillary components. Postoperatively, the patient received radioactive iodine ablation therapy (iodine-131) and a course of external beam radiation therapy to the sacroiliac area and other metastatic regions. No recurrences were observed in a follow-up period of 5 years after surgery.

Conclusion: The metastasis of differentiated thyroid carcinoma as a component of PDTC to the intranasal cavity has not been reported before. It is interesting that the well-differentiated component of the tumor was metastasized in our patient. Due to the aggressiveness of PDTC and the poor survival rates in patients who undergo surgery alone, a multidisciplinary treatment approach is required.

Keywords: Metastasis, nasal cavity, papillary thyroid carcinoma, thyroid neoplasm
Poorly differentiated thyroid carcinomas (PDTCs) account for only $4-7 \%$ of all thyroid carcinomas (1). PDTCs lie, both morphologically and behaviorally, between well-differentiated and undifferentiated carcinomas. Thyroid carcinoma was first described in 1907 by Langhans (2), and PDTCs were described in 1983 (3). PDTCs originate from either follicular or papillary epithelial cells, and typically reveal a trabecular, insular, and/or solid histomorphological pattern (4).
Tumors in the other sites of the body rarely metastasize to paranasal sinuses. In a review, it was reported that the most common site of metastasis in the paranasal sinuses was maxilla followed by ethmoids, frontal sinus, and sphenoid; and most common primary tumors that metastasized to paranasal sinuses were kidney tumors, followed by lung, urogenital tract, breast, gastrointestinal tract, and thyroid tumors (5). 
In this case report, we present an intranasal metastasis of the papillary component of PDTC in a 48-year-old male for the first time in the literature.

\section{CASE PRESENTATION}

A 48-year-old male patient presented with massive epistaxis and nasal obstruction. On nasal examination, a bleeding, vascular mass was seen filling the left nasal cavity. Computed tomography (CT) and magnetic resonance imaging (MRI) of the paranasal sinuses (PNS) revealed a polypoid soft-tissue mass located in the left nasal cavity. The mass expanded and obliterated the nasal cavity (Figure 1). Angiography was performed due to the vascularity of the mass and the presence of massive epistaxis. On angiography, a 2 x $3 \mathrm{~cm}$-sized hypervascular mass supplied by the maxillary artery was seen in the left nasal cavity, and it was embolized. The patient underwent left medial maxillectomy, with an initial diagnosis of vascular malignancy (Figure 2). Histopathological examination showed neoplastic cells and immunohistochemical analysis was strongly positive for thyroglobulin. On microscopy, follicle-like structures were seen which were lined with a single row of epithelial cells with vesicular nuclei. The histopathological report was welldifferentiated thyroid carcinoma metastasis with a follicular pattern (Figure 3a, b). Whole body scintigraphy, ultrasonography and positron emission tomography (PET) were done to rule out other possible metastases in the body and determine the origin of the tumor. Thyroid ultrasonography revealed a 33 x $23 \mathrm{~mm}$, heterogeneous, hypoechoic nodule in the left lobe of the thyroid. In whole body scintigraphy and PET CT, the origin of the tumor was determined as the left lobe of the thyroid gland, and there were multiple metastases involving the lung, sacroiliac area, and left humerus. There was no regional lymph node metastasis. The patient underwent total thyroidectomy. Histopathological examination of the thyroidectomy specimen revealed PDTC consisting of insular, follicular, and papillary components. On histopathological examination, it was seen that tumor of our patient had mitotic activity, invaded the capsule of the tumor as well as the blood vessels, and formed solid tumor clusters (Figure 3c, d).

Postoperatively, the patient received radioactive iodine ablation therapy with 200 milliCuries (mCi) of iodine-131. Then he underwent a course of external beam radiation therapy (with a total dose of $2000 \mathrm{cGy}$ ) to the sacroiliac area and other metastatic regions. No recurrences were observed in a followup period of 5 years after surgery. Written informed consent was obtained from the patient.

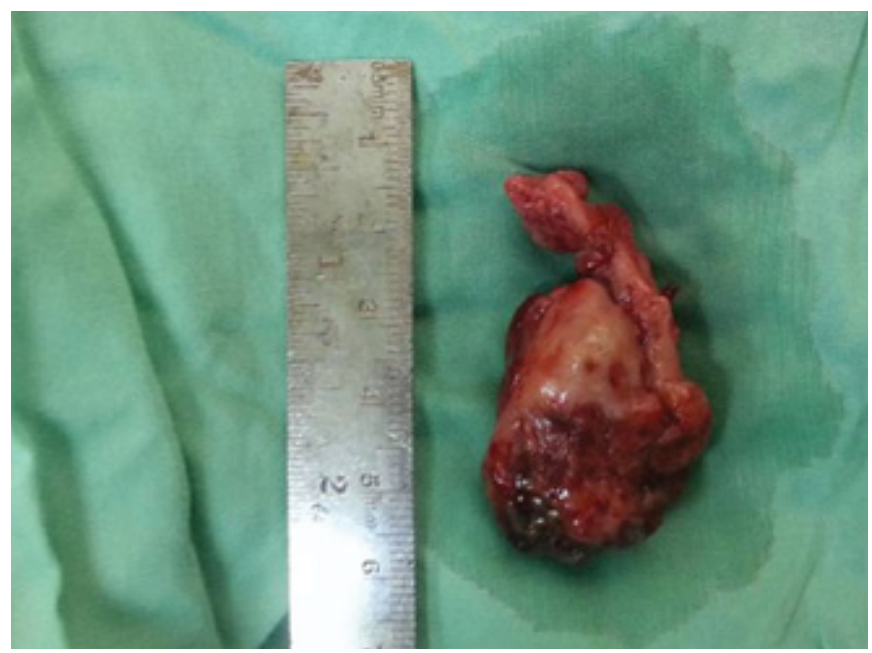

FIG. 2. Resected mass from the left nasal cavity
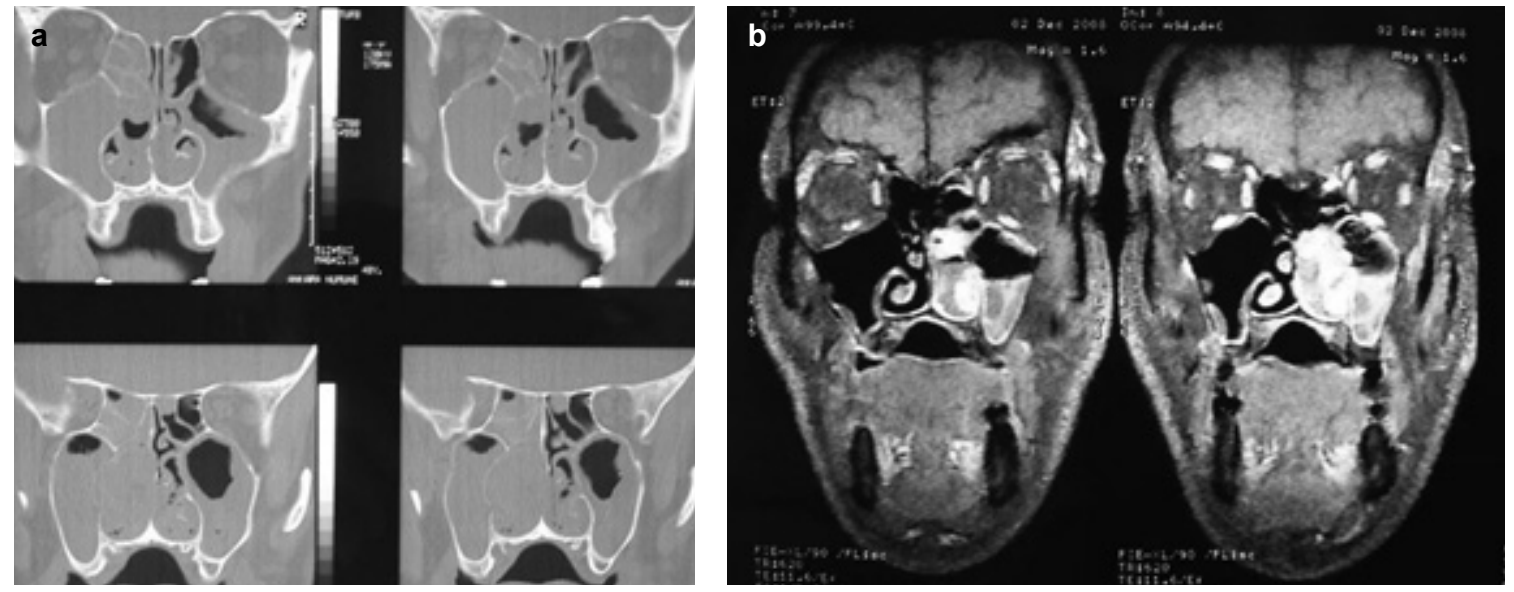

FIG. 1. a, b. Coronal computed tomography of the paranasal sinuses revealed a polypoid soft-tissue mass located in the left nasal cavity. It expanded and obliterated the nasal cavity (a). Coronal T1-weighted magnetic resonance imaging scan of the paranasal sinuses shows a mass filling the left nasal cavity (b). 

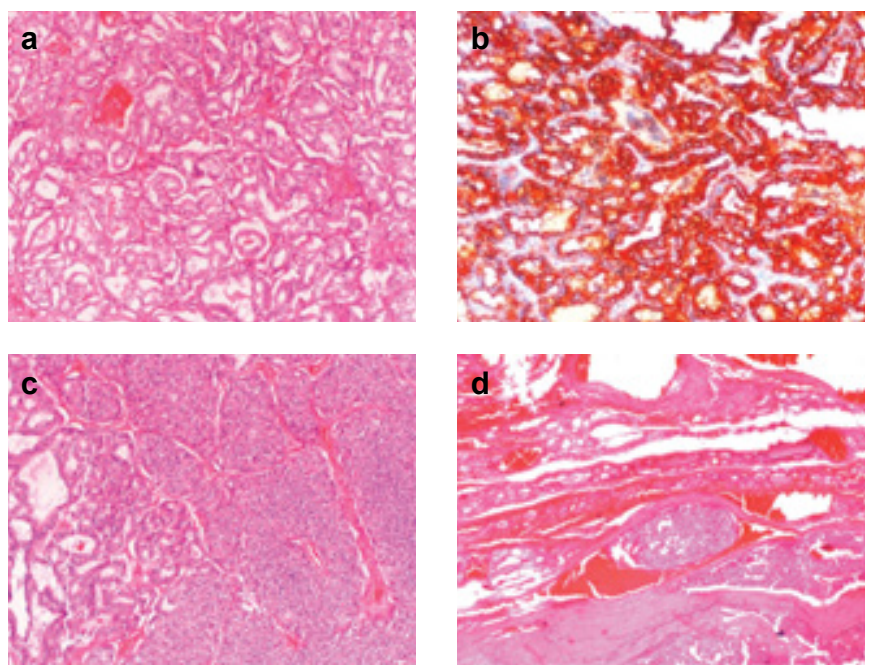

FIG. 3. a-d. Histopathological examination of the mass resected from the left nasal cavity shows neoplastic cells. Immunohistochemical analysis was strongly positive for thyroglobulin in the mass (a and b), and in thyroidectomy material (c and d); well-differentiated thyroid carcinoma metastases including a follicular pattern were detected. Follicle-like structures lined with a single row of epithelial cells with vesicular nuclei were noted (HE x 40) (a). Diffuse, strongly positive staining for thyroglobulin (X 100) (b). Insular pattern and well-differentiated areas are seen together in a focus. (HE x 40) (c). Capsular and vascular invasion (HE x 40) (d).

\section{DISCUSSION}

Despite the fact that most cases of well-differentiated thyroid carcinoma (WDTC) have excellent prognosis, patients with anaplastic thyroid carcinoma (ATC) have poor prognosis (4). PDTC is a rarely seen malignant thyroid tumor. When compared to WDTC and ATC, it behaves more aggressively than WDTC, however it is less destructive than undifferentiated thyroid carcinoma and ATC. More than 150 PDTC patients have been reported up to date (6).

Histopathological characteristics of PDTCs may reveal a solid, trabecular, or insular growth pattern. They do not have the histopathological features or classical nuclear features of papillary thyroid carcinoma, do not invade blood vessels or tumor capsule, and have either tortuous nuclei or necrosis or mitotic activity. In absence of trabecular or insular growth pattern, PDTC is not diagnosed. PDTC is excluded if tumor shows those characteristics, and principal nuclear characteristics of papillary thyroid carcinoma (PTC). In case of absence of histopathological features of PTC, tumor must be evaluated for presence of either tortuous nuclei, necrosis or mitotic activity, and in case of presence of any of those characteristics, it is diagnosed as PDTC. The lesion is thought to bea well-differentiated follicular thyroid carcinoma it hose features are not present (4).

PDTCs have a propensity to metastasize to regional lymph nodes (50-85\%) and to distant sites (3-85\%), most commonly to the lungs (14-54\%) and bones (18-33\%) (7). The 5- and 10 -year survival rates are considerably worse in patients with PDTC (50\% and 34\%, respectively) compared to patients with WDTC $(95 \%$ and $86 \%)(8)$.

Metastatis to the sinonasal tract is extremely rare, and metastatic tumors may be present with epistaxis, facial swelling, pain or nasal obstruction. In a literature review, Altman described 11 cases of metastatic thyroid cancers to PNS (9). Duque-Fisher et al. (10) reported two patients with metastatic carcinoma to the sphenoid sinus from the thyroid and pancreas, and two patients with metastatic renal cell carcinoma to the left nasal vestibule. Epistaxis was the most common presenting symptom, which is not surprising considering the hypervascular nature of thyroid tumors (9).

Sabih et al. (8) reported a patient with PDTC invading the sternum. The patient had a total thyroidectomy and resection of the manubrium with sternal reconstruction combined with adjuvant radioactive iodine ablation and external beam radiation therapy, and survived after 5 years despite a small local recurrence.

Our case had lung, paranasal sinus and bone (sacroiliac and humerus) metastases of PDTC that originated from the thyroid gland. Angiosarcoma, hemangioma or other vascular malignant tumors were initially considered in the differential diagnosis of the patient, so we did not perform a biopsy of the nasal mass before the medial maxillectomy so as not to cause massive bleeding. Treatment of the patient included left medial maxillectomy for resection of the mass in the left nasal cavity, total thyroidectomy, radioactive iodine therapy and external beam radiation radiotherapy for the sacroiliac area, humerus and lung. He had no evidence of tumor recurrence and/or residual tumor after 5 years.

Intranasal metastasis of thyroid carcinomas is very rare, and the primary tumor is usually WDTC. Treatment of choice is total thyroidectomy and cervical lymph node dissection. There is not enough data on postoperative radioactive iodine ablation, external radiation, or chemotherapy. Metastasis of differentiated thyroid carcinoma as a component of PDTC to the intranasal cavity has not been reported before. It is interesting that the well-differentiated component of the tumor was metastasized in our patient. Due to the aggressiveness of PDTC and the poor survival rates in patients who undergo surgery alone, a multidisciplinary treatment approach is required.

\section{Ethics Committee Approval: N/A.}

Informed Consent: Written informed consent was obtained from the patient.

Peer-review: Externally peer-reviewed. 
Author contributions: Concept - R.O.K., E.A., B.T.S.; Design - R.O.K., E.A., C.Ö.; Supervision - R.O.K., E.A., C.Ö.; Resource - R.O.K.; Materials - R.O.K., P.B., B.T.S.; Data Collection \&/or Processing - R.O.K., P.B.; Analysis \&/or Interpretation - R.O.K., C.Ö., E.A.; Literature Search - R.O.K., B.T.S.; Writing - R.O.K., E.A., B.T.S.; Critical Reviews - R.O.K., C.Ö., P.B.

Conflict of Interest: No conflict of interest was declared by the authors.

Financial Disclosure: The authors declared that this study has received no financial support.

\section{REFERENCES}

1. Rocha AS, Soares P, Fonseca E, Cameselle-Teijeiro J, Oliveira MC, Sobrinho-Simoes M. E-cadherin loss rather than betacatenin alterations is a common feature of poorly differentiated thyroid carcinomas. Histopathology 2003;42:580-7. [CrossRef]

2. Langhans T. Über die epithelialen Formen der malignen Struma. Virchows Archive 1907;189:69-152. [CrossRef]

3. Sakamoto A, Kasai N, Sugano H. Poorly differentiated carcinoma of the thyroid. A clinicopathologic entity for a highrisk group of papillary and follicular carcinomas. Cancer 1983;52:1849-55. [CrossRef]
4. Volante M, Collini P, Nikiforov YE, Sakamoto A, Kakudo K, Katoh R, et al. Poorly differentiated thyroid carcinoma: the Turin proposal for the use of uniform diagnostic criteria and an algorithmic diagnostic approach. Am J Surg Pathol 2007;31:125664. [CrossRef]

5. Bernstein JM, Montgomery WW, Balogh K, Jr. Metastatic tumors to the maxilla, nose, and paranasal sinuses. Laryngoscope 1966;76:621-50. [CrossRef]

6. Lam KY, Lo CY, Chan KW, Wan KY. Insular and anaplastic carcinoma of the thyroid: a 45-year comparative study at a single institution and a review of the significance of p53 and p21. Ann Surg 2000;231:329-38. [CrossRef]

7. Sanders EM, Jr., LiVolsi VA, Brierley J, Shin J, Randolph GW. An evidence-based review of poorly differentiated thyroid cancer. World J Surg 2007;31:934-45. [CrossRef]

8. Sabih Q, Spafford MF, Dietl CA. Poorly differentiated thyroid carcinoma with sternal invasion. A case report and review of the literature. Int J Surg Case Rep 2014;5:816-20. [CrossRef]

9. Altman KW, Mirza N, Philippe L. Metastatic follicular thyroid carcinoma to the paranasal sinuses: a case report and review. $J$ Laryngol Otol 1997;111:647-51. [CrossRef]

10. Duque-Fisher CS, Casiano R, Velez-Hoyos A, Londono-Bustamente AF. [Metastasis to the sinonasal region]. Acta Otorrinolaringol Esp 2009;60:428-31.[CrossRef] 